\title{
Evaluation of the Tissue Toxicity of Antiseptics by the Hen’s Egg Test on the Chorioallantoic Membrane (HETCAM)
}

\author{
C. Marquardt ${ }^{1 *}$, C. Matuschek ${ }^{2 *}$, E. Bölke², P. A. Gerber ${ }^{3}$, M. Peiper ${ }^{4}$, J. v. Seydlitz-Kurzbach ${ }^{5}$, \\ B. A. Buhren ${ }^{6}$, M. van Griensven ${ }^{7}$, W. Budach ${ }^{2}$, M. Hassan ${ }^{6}$, G. Kukova ${ }^{6}$, R. Mota ${ }^{6}$, D. Höfer $^{5 *}$, \\ K. Orth ${ }^{8 *}$,W. Fleischmann ${ }^{2 *}$ \\ ${ }^{1}$ Department of General and Visceral Surgery, Klinikum Ludwigsburg, Germany \\ 2Department of Radio oncology, University Hospital Düsseldorf, Germany \\ ${ }^{3}$ Physiology and Biophysics, College of Medicine, University of California, Irvine, USA \\ ${ }^{4}$ Department of Surgery, University Hospital Düsseldorf, Germany \\ ${ }^{5}$ Institute for Hygiene and Biotechnology, Hohenstein Research Institutes, Boennigheim, Germany \\ ${ }^{6}$ Department of Dermatology, University Hospital Düsseldorf, Germany \\ ${ }^{7}$ Ludwig Boltzmann Institute for Experimental and Clinical Traumatology, Research Center AUVA, Austrian Cluster for Tissue \\ Regeneration, Wien, Austria \\ ${ }^{8}$ Department of Surgery, Krankenhaus Hannover, Germany
}

\begin{abstract}
Background: Antiseptics are frequently used for the prophylaxis and treatment of local infections of chronic wounds. Whereas local antiseptics in general have a positive effect on wound healing an uncritical use may impair wound healing due to toxic side effects.

Objective: We sought to assess the vascular irritation potential of different antiseptic solutions and ointments commonly used for short and long term application as a measure of tissue toxicity.

Method: The vascular irritation was evaluated by the hen's egg test (HET) on the chorioallantoic membrane (CAM). The effects on the vessels of a mucous membrane were directly assessed by stereomicroscopic observation in vivo.

Results: Severe CAM irritation was observed after short-term applications of $1 \%$ octenidin- $2 \mathrm{HCl}$ (Octenisept $\left.^{\mathrm{TM}}\right), 72 \%$ isopropanol $\left(\right.$ Cutasept $\left.^{\mathrm{TM}}\right), 0.35 \%$ chloroxylenol (Dettol ${ }^{\mathrm{TM}}$ ) and 10\% PVP-I ointment (Betaisodona $^{\text {TM}}$ ). Medium irritations were observed for 10\% PVP-I solution (Betaisodona ${ }^{\mathrm{TM}}$ ), 3\% lysosomal PVP-I ointment (Repithel ${ }^{\mathrm{TM}}$ ), 1.8\% cadexomer-iodine ointment (Iodosorb ${ }^{\mathrm{TM}}$ ) and 1\% cadexomer-iodine pellets (Iodosorb ${ }^{\mathrm{TM}}$ ). Finally, slight irritations were observed for 1\% PVP-I solution (Betaisodona ${ }^{\mathrm{TM}}$ ), $0.1 \%$ polyhexanid plus betain (Prontosan ${ }^{\mathrm{TM}}$ ) and 1\% silversulfadiazine ointment (Flammazine ${ }^{\mathrm{TM}}$ ), whereas $0.04 \%$ polyhexanid solution (Lavanid $^{\mathrm{TM}}$ ), washings from sterile maggots of Lucilia sericata and filtrated enzymes from Clostridium histolyticum (Iruxol-NTM) showed no effects of irritation. In the long-term approaches, no vascular irritations were found for polyhexanid, washings from Lucilia sericata and enzyme filtrations from Clostridium histolyticum.

Conclusion: The vascular injuries caused by the studied
\end{abstract}

\footnotetext{
* Equal contribution
}

antiseptics are an indirect indicator of their tissue toxicity. Strikingly, even therapeutic substances, which have been regarded as safe in their application for the treatment of chronic wounds in clinical studies, showed severe irritations on the CAM. We suggest that agents with no or low irritation potential on the CAM should be preferred in the clinical practice in order to obtain optimal results.

Key words: vascular irritation, chronic wound, wound management, infection

\section{INTRODUCTION}

Antiseptic solutions and ointments are used for the prophylaxis (i.e. preoperative disinfections as short term application) and treatment of local infections or critical colonization of chronic wounds (long term application) [1-3]. In fact, the presence of large numbers of bacteria is known to decrease or even stop the process of normal wound healing. Indications for the application of antiseptics include not only clinical signs of local infections of acute or chronic wounds, but also critical bacterial colonization's of $>10^{5}$ colony forming units (cfu) per $\mathrm{g}$ of tissue, equaling $10^{3} \mathrm{cfu}$ per $\mathrm{ml}$ suspension in microbial swabs [4-6]. A recent multicenter study of 1000 wounds showed a general positive effect of local antiseptics on the wound healing process [27]. In contrast, a general uncritical use of antiseptics may lead to formation of resistance and even to a decrease of wound healing due to toxic side effects $[3,6,7]$.

In the clinical practice local therapeutics are differentiated according to their mode of application (short term and long term) [8-13]. Solutions are used for rinsing and cleaning of acute and chronic wounds in the short term application mode or in the long term application mode as a wet-to-dry non adherent wound dressing combined i.e. with gauze. Accordingly, oint- 
ments are used widely besides the broad spectrum of other modern wound therapeutics and dressings (i.e. nonadherent gauze dressings) in the long term therapy of acute or chronic wounds.

Main medical requirements for antiseptic preparations include a broad-spectrum of antimicrobial activity, a minor systemic toxicity and excellent local tissue compatibility $[1,14,15]$. The toxicity of local antiseptic agents including their injury of the granulation tissue is well investigated [5, 15-18]. Strikingly, a destructive effect on the vessels of the dense capillary plexus of the newly formed granulation tissue is regarded as one of the most severe potential damages caused by these preparations. Therefore, the indication for local antiseptics should be carefully assessed [4, 19].

Here, we used the in vivo hens' egg chorioallantoic membrane (CAM) test of chicken embryos as a model for the capillary plexus of the granulation tissue to analyse the tissue compatibility of the most commonly used antiseptics $[3,15,20,21]$. Vascular injuries to the CAM were regarded as measure for vascular irritation and hence tissue damage.

\section{Materials AND Methods}

\section{Tested Preparations}

The following test preparations were used for the HETCAM test: Ringer solution (B. Braun Melsungen AG, Melsungen, Germany), physiological sodium chloride solution $(0.9 \% \mathrm{NaCl}, \mathrm{B}$. Braun Melsungen AG, Melsungen, Germany) and phosphate-buffered saline (PBS, Fluka, Buchs, Switzerland) served as negative controls, 1\% sodium dodecyl sulfate (Fluka, Buchs, Switzerland) in PBS served as positive control. Furthermore, the following commercially available antiseptics were used: Cutasept ${ }^{\mathrm{TM}}$ (BODE Chemie Hamburg, Hamburg, Germany) containing $72 \%$ isopropanol, Octenisept ${ }^{\mathrm{TM}}$ (Schülke \& Mayr, Norderstedt, Germany) containing $0.1 \%$ octenidin- $2 \mathrm{HCl}$ plus $2 \%$ phenoxyethanol, Betaisodona ${ }^{\mathrm{TM}}$ solution (Mundipharma GmbH, Limburg/Lahn, Germany) containing 10\% povidone - iodine (PVP-I), Prontosan ${ }^{\mathrm{TM}}$ (Medical Instruments Corporation, Solothurn, Switzerland) containing $0.1 \%$ polyhexanid plus $0.1 \%$ undecyleneamidopropyl-betain, Lavanid ${ }^{\mathrm{TM}}$ (SERAG Wiessner KG, Naila, Germany) containing $0.04 \%$ polyhexanid, Flammazine $^{\mathrm{TM}}$ ointment (Solvay Arzneimittel $\mathrm{GmbH}$, Hannover, Germany) containing 1\% silver-sulfadiazine (SSD), Betaisodona ${ }^{\mathrm{TM}}$ ointment (Mundipharma GmbH, Limburg/Lahn, Germany) containing 10\% PVP-I, Repithel ${ }^{\mathrm{TM}}$ ointment (Mundipharma GmbH, Limburg/Lahn, Germany) containing $3 \%$ lysosomal PVP-I, Iodosorb ${ }^{\mathrm{TM}}$ ointment (Smith \& Nephew, Hull, UK) containing $1.8 \%$ cadexomer iodine and Iodosorb $^{\text {TM }}$ pellets containing 1\% cadexomer iodine. In addition we used freshly washed maggot secretions of Lucilia sericata (Neocura, Tübingen, Germany). Approximately two hundred 3 - 4 days old second instar maggots were washed with $20 \mathrm{ml} \mathrm{NaCl}$ solution $(0.9 \%)$ for $2 \mathrm{~h}$. Dettol $^{\mathrm{TM}}$ (R\&C Pharmaceuticals Ltd, Mobeni, RSA) an antiseptic solution widely used in Africa, containing 4.8\% chloroxylenol and $11.28 \%$ denaturated alcohol also was investigated. Prior to the application Dettol ${ }^{\mathrm{TM}}$ was diluted 1:14 with PBS like recommended by the manufacturer's instructions. This resulted in final concentrations of $0.35 \%$ chloroxylenol and $0.8 \%$ denaturated alcohol.

Finally, we used Iruxol-N ${ }^{\text {TM }}$ (Smith\&Nephew, Hull, UK), containing enzyme filtrations from Clostridium histolyticum (e.g. clostridiumpeptidase), as a non-antiseptic ointment for comparison to the tested antiseptic ointments. Iruxol-NTM is used for the enzymatic debridement of wounds.

\section{Chicken Embryo HETCAM System}

Fertile hens' eggs were taken form a local hatchery at day 8 of incubation at $37.5^{\circ} \mathrm{C}$. Eggs were removed from the incubator and swabbed with $70 \%$ ethanol. To ensure that the embryo was properly positioned, the eggs were left in a horizontal position for several minutes. After breaking up the shell, first all of the chorioallantoic membrane was evaluated using a stereo zoom microscope SZ11 (Olympus Optical, Hamburg, Germany) equipped with a digital camera (colour view III) and analysis software (Soft imaging system, Stuttgart, Germany). Only intact CAMs were used for the experiments.

\section{Semiquantitative Evaluation of the Vascular IRRITATION}

The evaluation of vascular irritation was determined after the official INVITTOX protocol No. 47 provided by the European Centre for the Validation of Alternative Methods (ECVAM)'s Scientific Information Service (SIS).

Following this protocol, $300 \mu \mathrm{l}$ of each test preparation were applied to the CAM. During the first 5 minutes (short term) and after 60 minutes (long term application), the vascular irritation was evaluated using the stereo microscope, which allows the direct assessment of erythrocytes passing through membrane capillaries. The occurrence of vascular haemorrhages, vascular lysis or vascular coagulations as morphological criteria in response to the test preparations were then used in the following formula:

$$
I S=\left[\frac{301-t(h)}{300}\right] * 5+\left[\frac{301-t(l)}{300}\right] * 7+\left[\frac{301-t(c)}{300}\right] * 9
$$

\section{Formula For the CALCUlation of the IrRitation SCORE (IS)}

The time $t$ in seconds marks the onset of the following effects during the first 5 minutes (300 seconds) of observation: $\mathrm{h}=$ vascular haemorrhage, $1=$ vascular lysis, $c=$ vascular coagulation, e.g. $\mathrm{t}(\mathrm{h})$ marks the onset time in seconds of vascular haemorrhage. The non-dimensional quotient is then multiplied by a factor indicating the impact on vascular damage of the observed effect, e.g. coagulation has the highest impact expressed by the multiplication factor 9. The IS comprehends values between 0 and 21

The effects of vascular irritation are measured semi-quantitatively and are differentiated in 4 IS ranges according to Spielmann. 

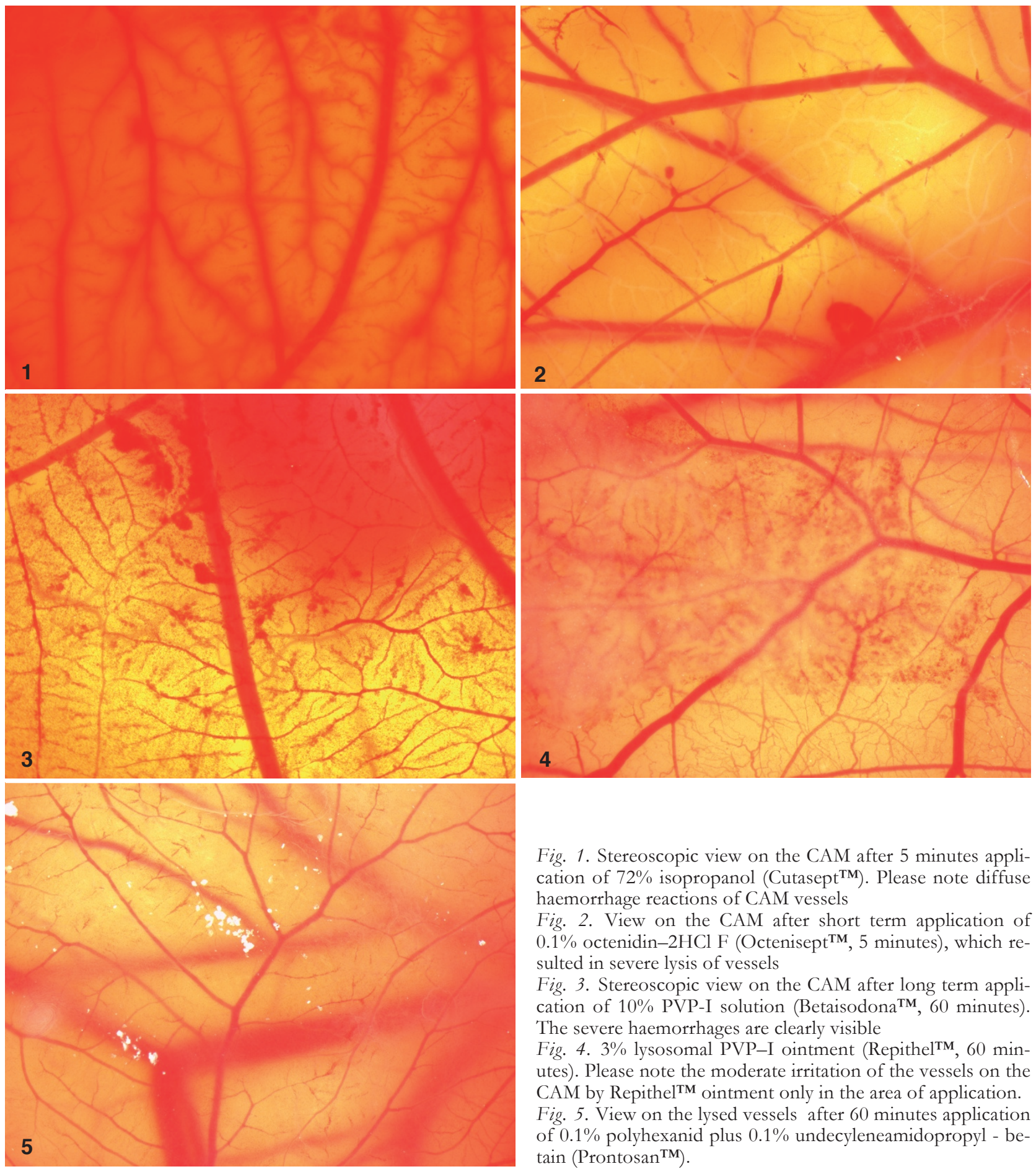

Fig. 1. Stereoscopic view on the CAM after 5 minutes application of $72 \%$ isopropanol (Cutasept ${ }^{\mathrm{TM}}$ ). Please note diffuse haemorrhage reactions of CAM vessels

Fig. 2. View on the CAM after short term application of $0.1 \%$ octenidin-2 $\mathrm{HCl} \mathrm{F}$ (Octenisept ${ }^{\mathrm{TM}}, 5$ minutes), which resulted in severe lysis of vessels

Fig. 3. Stereoscopic view on the CAM after long term application of 10\% PVP-I solution (Betaisodona ${ }^{\mathrm{TM}}$, 60 minutes). The severe haemorrhages are clearly visible

Fig. 4. 3\% lysosomal PVP-I ointment (Repithel ${ }^{\mathrm{TM}}$, 60 minutes). Please note the moderate irritation of the vessels on the CAM by Repithel ${ }^{\mathrm{TM}}$ ointment only in the area of application. Fig. 5. View on the lysed vessels after 60 minutes application of $0.1 \%$ polyhexanid plus $0.1 \%$ undecyleneamidopropyl - betain (Prontosan ${ }^{\mathrm{TM}}$ ).

$0=$ no reaction $(0.0-0.9)$

$1=$ slight reaction $(1.0-4.9)$

$2=$ moderate reaction $(5.0-8.9)$

$3=$ severe reaction $(9-21)$.

The irritation threshold (IT) expresses the toxicity level of a substance and is assessed by a dilution experiment on 3 eggs. After progressive dilution, the IT is defined as the highest concentration causing slight CAM irritation.

For each substance 3 eggs were observed continuously over 5 minutes to evaluate the IS, which was expressed as the mean value of the 3 experiments. In

order to evaluate the irritation-threshold, an additional 3 eggs were observed under application of different dilutions of the various substances tested.

For non-transparent antiseptic solutions and ointments the test was modified. Briefly, these substances were applied in thin layers $(<300 \mu \mathrm{L})$ which allowed vessel observation.

\section{RESULTS}

After application of different substances the observed reactions on the CAM were expressed as irritation scores (IS) and irritation thresholds (IT) according to 
Table 1. Irritation scores (IS), their standard deviation (STD) and irritation thresholds (IT) of antiseptics. IS Lit. = irritation scores of former studies.

\begin{tabular}{|c|c|c|c|c|c|}
\hline $\begin{array}{l}\text { Product } \% 0 \\
\text { negative controls }\end{array}$ & Substance & \multicolumn{2}{|c|}{ IS MV (STD) } & \multirow[t]{3}{*}{ IS Lit. 15, 16} & \multirow[t]{3}{*}{ IT } \\
\hline Rinsing solutions & $0,9 \%$ physiological saline, Ringer solution & 0.0 & $(0.0)$ & & \\
\hline $\begin{array}{l}\text { Buffered solution } \\
\text { positive controls }\end{array}$ & Phosphate-buffered saline & 0.0 & $(0.0)$ & & \\
\hline $\begin{array}{l}1 \% \text { SDS } \\
\text { solutions }\end{array}$ & Sodium dodecyl sulfat & 16.9 & $(1.5)$ & & \\
\hline Dettol $^{\mathrm{TM}} 1: 14$ & $0.35 \%$ chloroxylenol $+0.8 \%$ denaturated alcohol & 17.4 & $(0.0)$ & & $1: 500$ \\
\hline Cutasept ${ }^{\mathrm{TM}}$ & $72 \%$ isopropanol & 18.2 & $(0.8)$ & 18.0 & $1: 200$ \\
\hline Octenisept $\mathrm{t}^{\mathrm{TM}}$ & $0.1 \%$ octenidin $-2 \mathrm{HCl}$ & 14.8 & $(5.1)$ & 14.0 & $1: 100$ \\
\hline Betaisodona $^{\mathrm{TM}}$ & $10 \%$ PVP-I & 5.3 & $(1.6)$ & & $1: 10$ \\
\hline Betaisodona $^{\mathrm{TM}}$ 1:10 & $1 \%$ PVP-I & 1.3 & $(0.8)$ & 2.0 & \\
\hline Prontosan $^{\mathrm{TM}}$ & $0.1 \%$ polyhexanid & 1.6 & $(2.7)$ & & \\
\hline Lavanid $^{\mathrm{TM}}$ & $0.04 \%$ polyhexanid & 0.0 & $(0.0)$ & 0.0 & \\
\hline MDT & sterile washings from Lucilia sericata maggots & 0.0 & $(0.0)$ & & \\
\hline \multicolumn{6}{|l|}{ Ointments, pellets } \\
\hline Iruxol-NTM & Enzyme filtrations from Clostridium histolyticum & 0.0 & $(0.0)$ & & \\
\hline Flammazine $^{\mathrm{TM}}$ & $1 \%$ silver-sulfadiazine & 1.3 & $(2.2)$ & & \\
\hline Betaisodona ${ }^{\mathrm{TM}}$ & $10 \%$ PVP-I & 15.8 & $(2.1)$ & & \\
\hline Repithel $^{\mathrm{TM}}$ & 3\% lysosomal PVP-I & 4.8 & $(2.1)$ & & \\
\hline Iodosorb $^{\mathrm{TM}}$ ointment & $1,8 \%$ cadexomer-iodine & 8.3 & $(3.5)$ & & \\
\hline Iodosorb $^{\mathrm{TM}}$ pellets & $1 \%$ cadexomer-iodine & 5.7 & $(3.5)$ & & \\
\hline
\end{tabular}

Spielmann. Additionally, we included irritation scores of former studies [8, 16]. Ringer solution, $0.9 \%$ sodium chloride solution $(\mathrm{NaCl})$ and phosphate-buffered saline solution (PBS) did not show any effect on the vessels of the CAM (IS $=0.0$, STD $=0.0$ ) even in the long term mode of application after 60 minutes, as expected. Contrarily, the positive control using $1 \%$ sodium dodecyl sulfate (SDS) caused severe irritations on the CAM (IS = 16.9, STD = 1.5), (data not shown).

In the group of solutions the local antiseptics $\mathrm{Cu}$ tasept $^{\mathrm{TM}}$ (72\% isopropanol, IS $=18.2$, STD $\left.=0.8\right)$, Octenisept $\mathrm{t}^{\mathrm{TM}}(0.1 \%$ octenidin $-2 \mathrm{HCl}$ plus $2 \%$ phenoxyethanol, IS $=14.8$, STD $=5.1$ ) and Dettol ${ }^{\mathrm{TM}}$ $(0.35 \%$ chloroxylenol and $0.8 \%$ denaturated alcohol, IS $=17.4$, STD $=0.0$ ) caused severe irritations (Fig. 1-2).

Betaisodona $^{\mathrm{TM}}$ solution $(10 \%$ PVP-I, IS $=5.3$, STD 1.6) showed moderate effects in the short-term application, whereas a 1:10 dilution with PBS (1\% PVP-I, $\mathrm{IS}=1.3, \mathrm{STD}=0.8)$ only resulted in slight vascular damage. In the long-term approach, Betaisodona ${ }^{\mathrm{TM}}$ solution caused severe reactions of the CAM (Fig. 3). Prontosan ${ }^{\mathrm{TM}}$ wound rinsing solution $(0.1 \%$ polyhexanid plus $0.1 \%$ undecyleneamidopropyl betain, IS $=1.6$, STD $=2.7$ ) displayed only slight effects in the shortterm application. Similarly to Betaisodona, long-term use of this substance led to severe vascular irritation, mainly through vessel lysis (Fig. 5).

Lavanid $^{\mathrm{TM}}(0.04 \%$ polyhexanid, IS $=0.0$, STD $=$ $0.0)$ and the secretions from sterile maggots of Lucilia sericata (IS $=0.0$, STD $=0.0$ ) did not cause irritative effects even after long-term application. The irritation-threshold (dilution with PBS, at which only slight effects occurred on the CAM of 3 different eggs) was determined for those solutions with high irritative potential: Cutasept ${ }^{\mathrm{TM}}$, Dettol ${ }^{\mathrm{TM}}$, Octenisept ${ }^{\mathrm{TM}}$ and Betaisodona $^{\mathrm{TM}}$. To achieve a slight IS, Dettol ${ }^{\mathrm{TM}}$ had to be diluted to $1: 500$, Cutasept ${ }^{\mathrm{TM}}$ to $1: 200$, Octenisept $^{\mathrm{TM}}$ to $1: 100$ and Betaisodona ${ }^{\mathrm{TM}}$ to $1: 10$ (with a final PVP-Iodine concentration of $1 \%$ ).

In the group of ointments, Betaisodona ${ }^{\mathrm{TM}}(10 \%$ PVP-I, IS $=15.8$, STD $=2.1$ ) showed severe vascular irritations. Repithel ${ }^{\mathrm{TM}}$ (3\% lysosomal PVP-I, IS $=4.8$, STD $=2.1)$, Iodosorb ${ }^{\mathrm{TM}}$ ointment $(1.8 \%$ cadexomeriodine, IS $=4.8$, STD $=2.1$ ) and Iodosorb ${ }^{\mathrm{TM}}$ pellets $(1 \%$ cadexomer-odine, IS $=5.7$, STD $=3.5)$ displayed moderate irritations on the CAM in the short term and in the long-term application (Fig. 4).

Finally, Flammazine $^{\mathrm{TM}}$ (1\% silver-sulfadiazine, IS = 1.3, STD $=2.2$ ) showed only slight irritation, whereas Iruxol-NTM (enzyme from Clostridium histolyticum, IS $=0.0$, STD $=0.0)$ displayed no visible effect on the CAM.

Irritation scores and irritation thresholds of antiseptics and results for long-term applications are summarized in Table 1 and 2. Notably, only specimens showing no, slight or moderate irritation after 5 minutes exposure were tested for 60 minutes. Strikingly, solely $0.04 \%$ polyhexanid (Lavanid) and maggot secretions showed no irritation in the studied model. Iodine preparations with low iodine content (Repithel ${ }^{\mathrm{TM}}$ and Iodosorb ${ }^{\mathrm{TM}}$ ) resulted in moderate vascular irritations on the CAM. Furthermore, Iruxol-N ${ }^{\mathrm{TM}}$ showed no vascular irritations and effects were comparable to the negative control in the group of ointments. 
Table 2. Angioirritative potential of antiseptics in long term application.

\begin{tabular}{|c|c|c|c|}
\hline \multicolumn{4}{|l|}{ negative controls } \\
\hline Rinsing solutions & $0,9 \%$ physiological saline, Ringer solution & no & 60 \\
\hline \multicolumn{3}{|l|}{ positive control } & 60 \\
\hline \multicolumn{4}{|l|}{ solutions: } \\
\hline Dettol $^{\mathrm{TM}}$ & $0.35 \%$ chloroxylenol $+0.8 \%$ denaturated alcohol & severe & 5 \\
\hline Cutasept ${ }^{\mathrm{TM}}$ & $72 \%$ isopropanol & severe & 5 \\
\hline Octenisept ${ }^{\mathrm{TM}}$ & $0.1 \%$ octenidin $-2 \mathrm{HCl}$ & severe & 5 \\
\hline Betaisodona $^{\mathrm{TM}}$ & $10 \%$ PVP-I & severe & 60 \\
\hline Betaisodona $^{\mathrm{TM}}$ 1:10 & 1\% PVP-I & severe & 60 \\
\hline Prontosan ${ }^{\mathrm{TM}}$ & $0.1 \%$ polyhexanid & severe & 60 \\
\hline Lavanid ${ }^{\mathrm{TM}}$ & $0.04 \%$ polyhexanid & no & 60 \\
\hline MDT & sterile washings from Lucilia sericata maggots & no & 60 \\
\hline \multicolumn{4}{|l|}{ Ointments, pellets } \\
\hline Iruxol-NTM & Enzyme filtrations from Clostridium histolyticum & no & 5 \\
\hline Flammazine $^{\mathrm{TM}}$ & $1 \%$ silver-sulfadiazine & slight & 5 \\
\hline Betaisodona $^{\mathrm{TM}}$ ointment & $10 \%$ PVP-I & severe & 5 \\
\hline Repithel ${ }^{\mathrm{TM}}$ & 3\% lysosomal PVP-I & moderate & 60 \\
\hline Iodosorb $^{\mathrm{TM}}$ & $1,8 \%$ cadexomer-iodine & moderate & 60 \\
\hline Iodosorb $^{\mathrm{TM}}$ Pellets & $1 \%$ cadexomer-iodine & moderate & 60 \\
\hline
\end{tabular}

\section{Discussion}

In the present study we analysed the vascular irritation potential of the most commonly used antiseptic solutions and ointments for wound treatment [16, 20]. Additionally, we sought to compare traditional antiseptic formulations with modern concepts such as maggot debridement therapy. The HETCAM test - a method which evaluates morphological criteria such as haemorrhages, vascular lysis and coagulation - was chosen as a model for the evaluation of vascular irritation in wound granulation tissue $[8,16,20]$

Various reports have shown that commonly used antiseptics for wound treatment may cause vascular irritation at clinical concentrations $[2,15,22]$. Accordingly, our study demonstrated severe tissue toxicity for $72 \%$ isopropanol (IS $=18.2$ ), $0.35 \%$ chloroxylenol plus $0.8 \%$ denaturated alcohol $(1: 14$, IS $=17.4)$ and $0.1 \%$ octenidin-2HCl (IS = 14.8). Preparations containing PVP-I resulted have also presented milder but significant vascular damage. This is in line with investigations which have demonstrated that the prolonged exposure of human skin to aqueous iodine-solutions may cause irritations or rarely even severe skin reactions [5, 17, 20, 23-25]. Moreover, highly diluted PVPI-solutions were shown to bear severe cytotoxicity in cell cultures of human skin fibroblasts in vitro $[5,17$, 20, 24]. Notably, whereas Polyhexanid in a concentration of $0.04 \%$ caused no vascular damage, a $0.1 \%$ solution led to slight CAM irritation.

Stimulated by the recent progress in wound therapy, we included washings from Lucilia sericata, an organ- ism used in biological wound debridement and enzyme filtrations from Clostridium histolyticum (Iruxol-NTM) in our investigation. Both substances caused no CAM irritation in the studied model, suggesting good tolerability.

In order to avoid local toxic reactions, antiseptics are frequently diluted with physiological rinsing solutions, like physiological saline or Ringer solutions, e.g. $10 \%$ iodine is diluted 1:10 before wound treatment or abdominal rinsing [17]. Accordingly, the determination of potentially safe concentrations is of relevance to the clinical practice. Hence, we used rinsing solutions as well as dilutions of antiseptics to determine their irritation-thresholds (IT). Since IT evaluation is only possible for substances with moderate or severe reactions, we determined the IT for $72 \%$ isopropanol (IT $=1: 200), 0.1 \%$ octenidin-2HCl $(\mathrm{IT}=1: 100), 4.8 \%$ chloroxylenol plus $11.28 \%$ denatured alcohol (IT = 1:500) and 10\% PVP-I (IT = 1:10). Herein, 10\% PVPI, with an IT of 1:10, was the substance which showed the lowest toxic potential, whereas $4.8 \%$ chloroxylenol plus $11.28 \%$ denatured alcohol achieved the highest IT (1:500).

In a clinical setting, local antiseptics will first get in contact with fibrin, blood and debris. Beyond this surface, one can assume that either fresh granulation tissue or fibrotic tissue due to chronic impaired wound healing will be found [8]. Cytotoxic effects on this layer could be tolerated, if there was no damage on deeper structures like the vascular plexus [5]. Damage on the vascular plexus (haemorrhage, vascular lysis or coagulation) could result in severe impairment of tissue 
regeneration and poor or even no wound healing [26]. Furthermore, the cytotoxicity is concentration-dependent and correlates inversely with the complexity of the testing system. The development of antiseptics with low cytotoxic potential may improve wound healing through the preservation of the fragile neo-angiogenic vascular tissue.

We here provide further evidence for the toxicity of antiseptics commonly used in clinical practice. Our results indicate the good tolerability of low concentrated polyhexanid-solutions and biological wound treatments, as shown by HETCAM analyses.

The HETCAM test is a cheap and easy method to asses the vascular irritation potential of substances on a vascular system. Its relevance can be classified between in vivo and ex vivo test-systems. Comparing the different measured effects of the antiseptics used in this study with results from former clinical trials, a good accordance was found. We conclude that a medium irritation effect in the HETCAM can be regarded as safe for the application of antiseptic substances on chronic wounds, whereas substances with a severe irritation effect should be avoided in the clinical setting. For the therapy of chronic wounds we therefore recommend the use of $0.04 \%$ polyhexanid and maggot debridement. Beside this, 1\% silver-sulfadiazine and enzymatic filtrations from Clostridium histolyticum are regarded as safe. All tested iodine preparations with iodine concentrations up to $3 \%$ showed only moderate adverse effects and may therefore also be regarded safe for the treatment of chronic wounds.

\section{REFERENCES}

1. Bornside GH, Bornside BB. Comparison between moist swab and tissue biopsy methods for quantitation of bacteria in experimental incisional wounds. J Trauma 1979; 19: 103-105.

2. Brennan SS, Foster ME, Leaper DJ. Antiseptic toxicity in wounds healing by secondary intention. J Hosp Infect 1986; 8: 263-267.

3. Moller M, Suschke U, Nolkemper S et al. Antibacterial, antiviral, antiproliferative and apoptosis-inducing properties of Brackenridgea zanguebarica (Ochnaceae). J Pharm Pharmacol 2006; 58: 1131-1138.

4. Langer S, Sedigh Salakdeh M, Goertz O et al. The impact of topical antiseptics on skin microcirculation. Eur J Med Res 2004; 9: 449-454.

5. Muller G, Kramer A. Biocompatibility index of antiseptic agents by parallel assessment of antimicrobial activity and cellular cytotoxicity. J Antimicrob Chemother 2008; 61: 1281-1287.

6. Webster J, Osborne S. Preoperative bathing or showering with skin antiseptics to prevent surgical site infection. Cochrane Database Syst Rev 2006; CD004985.

7. Beam JW. Topical silver for infected wounds. J Athl Train 2009; 44: 531-533.

8. Gardner SE, Frantz RA, Doebbeling BN. The validity of the clinical signs and symptoms used to identify localized chronic wound infection. Wound Repair Regen 2001; 9: 178-186.

9. Marquardt C, Bolke E, Gerber PA et al. Correlation of cutaneous tension distribution and tissue oxygenation with acute external tissue expansion. Eur J Med Res 2009; 14: 480-486.

10. O'Meara S, Al-Kurdi D, Ologun Y, Ovington LG. Antibiotics and antiseptics for venous leg ulcers. Cochrane Database Syst Rev 2010; CD003557.
11. Barbaud A, Collet E, Le Coz CJ et al. Contact allergy in chronic leg ulcers: results of a multicentre study carried out in 423 patients and proposal for an updated series of patch tests. Contact Dermatitis 2009; 60: 279-287.

12. Ryssel H, Kloeters O, Germann G et al. The antimicrobial effect of acetic acid--an alternative to common local antiseptics? Burns 2009; 35: 695-700.

13. Mary Ammons CB. Anti-Biofilm Strategies and the Need for Innovations in Wound Care. Recent Pat Antiinfect Drug Discov 2009.

14. Fleischmann W, Russ M, Moch D, Marquardt C. [Biosurgery - maggots, are they really the better surgeons?]. Chirurg 1999; 70: 1340-1346.

15. Kalteis T, Luring C, Schaumburger J et al. [Tissue toxicity of antiseptics]. Z Orthop Ihre Grenzgeb 2003; 141: 233238.

16. Luepke NP. Hen's egg chorioallantoic membrane test for irritation potential. Food Chem Toxicol 1985; 23: 287291.

17. Reimer K, Vogt PM, Broegmann B et al. An innovative topical drug formulation for wound healing and infection treatment: in vitro and in vivo investigations of a povidone-iodine liposome hydrogel. Dermatology 2000; 201: 235-241.

18. Rizzi SC, Upton Z, Bott K, Dargaville TR. Recent advances in dermal wound healing: biomedical device approaches. Expert Rev Med Devices 2010; 7: 143-154.

19. Brennan SS, Leaper DJ. The effect of antiseptics on the healing wound: a study using the rabbit ear chamber. Br J Surg 1985; 72: 780-782.

20. Wutzler P, Sauerbrei A, Hartl A, Reimer K. Comparative testing of liposomal and aqueous formulations of povidone-iodine for their angioirritative potential at the chorioallantoic membrane of ex ovo cultivated chick embryos. Dermatology 2003; 207: 43-47.

21. Kishore AS, Surekha PA, Sekhar PV et al. Hen egg chorioallantoic membrane bioassay: an in vitro alternative to draize eye irritation test for pesticide screening. Int J Toxicol 2008; 27: 449-453.

22. Raahave D, Friis-Moller A, Bjerre-Jepsen $K$ et al. The infective dose of aerobic and anaerobic bacteria in postoperative wound sepsis. Arch Surg 1986; 121: 924-929.

23. Lamme EN, Gustafsson TO, Middelkoop E. Cadexomeriodine ointment shows stimulation of epidermal regeneration in experimental full-thickness wounds. Arch Dermatol Res 1998; 290: 18-24.

24. Steen M. Review of the use of povidone-iodine (PVP-I) in the treatment of burns. Postgrad Med J 1993; 69 Suppl 3: S84-92.

25. Viljanto J. Disinfection of surgical wounds without inhibition of normal wound healing. Arch Surg 1980; 115: 253-256.

26. O'Meara S, Cullum N, Majid M, Sheldon T. Systematic reviews of wound care management: (3) antimicrobial agents for chronic wounds; (4) diabetic foot ulceration. Health Technol Assess 2000; 4: 1-237.

27. Coerper S, Wicke C, Pfeffer F, Köveker G, Becker HD. Documentation of 7051 chronic wounds using a new computerized system within a network of wound care centers. Arch Surg. 2004 Mar;139(3):251-8.

Received: February 8, 2010 / Accepted: March 9, 2010

Address for correspondence:

PD. Dr. Edwin Bölke

Moorenstr. 5

40225 Düsseldorf

Germany

Phone: +49-211-81 17990

Fax: +49-211-81 18051

E-mail: boelke@med.uni-duesseldorf.de 